\title{
Pre-induced Lac Operon Effect on Non Specific Sugars: Pre-culture Effect is Dependent on Strength of Induction, Exponential Phase and Substrate Concentration
}

\author{
Pushkar Malakar* \\ Department of Bioscience \& Bioengineering, Indian Institute of Technology Bombay, Powai, Mumbai-400076, \\ Maharashtra, India
}

\begin{abstract}
The source and history of the cell plays an important role in influencing the phenotypic properties of the organism in a particular environmental condition. Pre-induced lac operon provides benefit on lactose environment. During metabolism lactose is broken down into glucose and galactose. The fate of cells with pre-induced lac operon on glucose and galactose milieu is not known. The influence of nutritional status of the medium, level of pre-induction and growth phase on pre-culture effect is not investigated. Effect of pre-induced lac operon on non specific sugars along with the factors that influence this effect was enumerated in the present study. Results of this present study indicate that preinduced lac operon provide benefit in terms of growth on galactose milieu. This study also suggests that Pre induced lac operon effect depends on the (i) strength of induction in the pre-culture, (ii) nutritional content of the environment and (iii) exponential growth phase of the organism. The above study will help in the better characterization of the pre culture effect. It will also help in the better understanding of the relation between gene expression and growth physiology.
\end{abstract}

Keywords: Entry into Early Exponential Phase, Galactose, IPTG and Strength of Induction, Late Exponential Phase, Preinduced lac operon.

\section{INTRODUCTION}

The source and history of the cell plays an important role in determining its fate $[1,2]$. Escherichia coli, cells, precultured on IPTG possess phenotypic growth advantages on lactose environment as compared to the ones which are not exposed to IPTG in pre-culture[3]. Such study has also been performed in GAL Regulon of Saccharomyces cerevisiae $[2]$.

Cells transferred from one medium to another medium of the same nutritional strength will show lower lag phase. If the media are distinctively different, there will be a higher lag phase. Exposure to IPTG in pre-culture provided phenotypic growth superiority to E.coli cells on lactose environment. This is due to the fact that cells require less time to adapt to the condition of the new environment exposed. It is not clear whether higher growth rate on lactose medium due to pre-exposure to IPTG is restricted only for lactose medium or is applicable to other carbon sources as well. IPTG activates lac operon [4]. $\beta$-galactosidase is produced on activation of lac operon. $\beta$-galactosidase cleaves lactose into glucose and galactose [5]. Thus, whether exposure to IPTG will provide any growth benefit to cells on glucose and galactose environment as compared to the ones which are not exposed to IPTG is an open question.

*Address correspondence to this author at the Biosciences and Bioengineering, Indian Institute of Technology Bombay, Powai, Mumbai400076, Maharashtra, India; Tel: +91-22-2576 4215; Fax: +91-22-2572 6895; E-mails: pushkarbt@iitb.ac.in; pushkarbt@gmail.com
The macromolecular content of the cell i.e. DNA, RNA and protein depends upon the growth rate of the organism [6]. The growth rate in turn is dependent on the nutritional status or strength of the medium. Nutritional strength refers to the ease with which building blocks of the cell i.e. amino acids are synthesized. Thus, the macromolecular content of the cell depends indirectly on the nutritional status of the milieu in which cells are growing.

Growth rate regulates gene expression [7, 8]. Several genes are upregulated or downregulated with increasing growth rate [9-13]. However, the impact of gene expression on growth rate is not clearly understood. Gene expression is a stochastic process [14]. Induction of lac operon by IPTG is concentration dependent. A critical concentration of IPTG is required for activation of the lac operon to its maximum strength. Impact of varied lac operon expression on the growth rate of an organism on lactose milieu is not known. Substrate plays an important role in determining the effect of gene expression [15]. It had been shown that substrate plays an important role in determining cost or the reduction in growth rate due to unnecessary gene expression [3, 16-18]. The effect of substrate on the phenotypic growth behavior of varied pre-induced lac operon on lactose environment is not known. Cost is shown to be dependent on different phases of exponential growth. Therefore the current study also explored the fate of cells with pre-induced lac operon in entry into Early Exponential Phase (EEP) and Late Exponential Phase (LEP) on lactose environment at low and high lactose concentrations [19]. 
In the present study, effect of pre-induced lac operon on the growth phenotype of E.coli cells on glucose and galactose milieu is looked upon. The study is further extended to see whether pre-culture on glucose or galactose will provide any growth benefit on lactose environment. E.coli cells were exposed to varied strength of IPTG in preculture and then their growth properties was checked on varied lactose concentration along with $\beta$-galactosidase measurement in EEP and LEP. This work will help in understanding the effect of cellular environment and its influence on growth. It will also help in the better characterization of the lac operon and how its benefit growth on lactose environment.

\section{MATERIALS AND METHODS}

\section{Strains, Media and Reagents}

E.coli MG1655(CGSC 6300) was used in the study [20]. All experiments were done in $\mathrm{M} 9$ defined medium consisting of M9 salts, $1 \mathrm{mM} \mathrm{MgSO} 4,0.1 \mathrm{mM} \mathrm{CaCl} 2$ with specified carbon source. Glycerol (Sigma), Galactose (Himedia), Glucose (Himedia), Lactose Monohydrate (Himedia) was used as carbon source. Z BUFFER $\left(60 \mathrm{mM} \mathrm{Na} \mathrm{mPO}_{4}, 40\right.$ $\mathrm{mM} \mathrm{NaH} \mathrm{PO}_{4}, 10 \mathrm{mM} \mathrm{KCl}, 1 \mathrm{mM} \mathrm{MgSO} 4,50 \mathrm{mM} 2-$ mercaptoethanol, pH 7.0). ONPG: $40 \mathrm{mg}$ ONPG dissolved in $10.0 \mathrm{ml}$ of $0.1 \mathrm{M}$ potassium phosphate buffer $\mathrm{pH}$ 7.0. IPTG (Invitrogen) was used for inducing the lac operon in pre-culture.

\section{Growth rate estimation}

The exponential growth rate of E.coli was measured by growing the strains in $50 \mathrm{ml}$ culture in $250 \mathrm{ml}$ flask. These flasks were incubated on shaker at 37 degree Celsius at 240 rpm. The pre-cultures were centrifuged to remove the entire initial pre-culture medium and resuspended in the new culture medium to calibrate the sample to inoculate into the experimental medium. The starting O.D. was almost similar
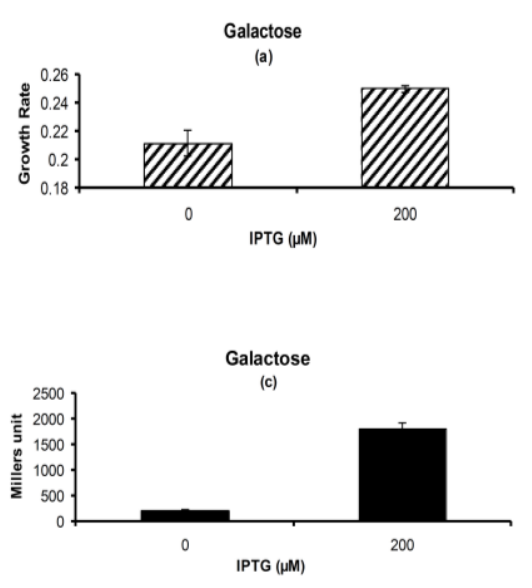

in all the cases. Samples were taken out frequently and O.D. was measured at $595 \mathrm{~nm}$ using ELISA Reader (BioRad). Entry into Early Exponential Phase refers to the growth of the cells in the initial 1 to 1.5 hours of the growth phase. Late Exponential Phase refers to the growth of the cells from 1.5 to 4.5 hours in the exponential phase. All the experiments were done in duplicates. Experimental parameters represent the average of three independent experiments.

\section{Beta Galactosidase Protocol}

Cells were grown on M9 medium with different carbon sources. Fixed Aliquots of culture were taken at different O.D. in $2 \mathrm{ml}$ eppendorf tube. Centrifuged and resuspended in $1 \mathrm{ml}$ Z-buffer. O.D. was measured at $595 \mathrm{~nm} 80 \mu \mathrm{l}$ of $0.1 \%$ SDS and $160 \mu \mathrm{l}$ of chloroform were added to each tube. The tubes were vortexed for 15 seconds. The reaction mixture was incubated at 30 degree Celsius for 15 minutes. $160 \mu \mathrm{l}$ of $4 \mathrm{mg} / \mathrm{ml}$ ONPG was then added and vortexed well for $10 \mathrm{sec}$. The reaction tubes were incubated at 30 degree Celsius for 10 minutes. The reaction was quenched by adding $400 \mu \mathrm{l}$ of $1 \mathrm{M}$ sodium carbonate. Cell debris was spinned down. O.D. was measured at $420 \mathrm{~nm}$. Miller Units was calculated using the following formula: $U=1000 \times[(O D 420) /[($ Time $) \times$ (Vol) x OD595] Where Vol is volume of culture used in the assay in mls, and Time is in minutes incubated after adding ONPG at 30 degree $\mathrm{C}$.

\section{RESULTS}

The focus of the study was to characterize the effect of pre-induced lac operon on glucose and galactose milieu along with the factors that influence the effect of pre-induced lac operon. Cells previously exposed to IPTG along with unexposed cells were grown on galactose medium. It was observed that cells which are exposed to IPTG showed higher growth rate as compared to the ones which are not exposed to IPTG (Fig 1a). The percentage relative reduction in growth rate or burden for the cells which were not pre-

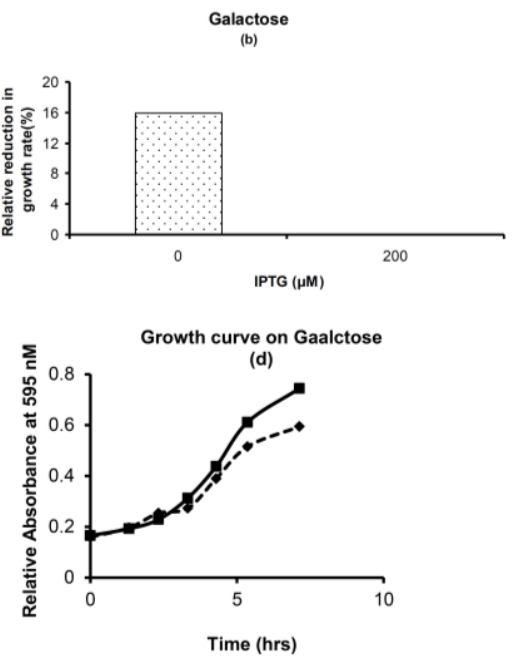

Fig. (1). Phenotypic Growth Properties on Galactose: (a) Growth rate for cells with and without pre-exposure to IPTG. (b) Percentage Relative reduction in growth rate for cells which are not exposed to IPTG in pre-culture as compared to the ones which are exposed to IPTG. (c) $\beta$-galactosidase activity for cells which are exposed to IPTG or not in pre-culture. (d) Growth Curve. Solid Line with square represents the cells which are exposed to $200 \mu \mathrm{M}$ of IPTG in M9 glycerol medium in pre-culture. Dashed Line with diamond represents the cells which are not exposed to IPTG in M9 glycerol medium in pre-culture. Glycerol $(1 \mathrm{~g} / \mathrm{L})$ and Galactose $(10 \mathrm{~g} / \mathrm{L})$ was used in experiment. 
exposed to IPTG in pre-culture was around $15 \%$ as compared to the ones which were exposed (Fig. 1b). The cells were subjected for $\beta$-galactosidase measurement on galactose environment. The $\beta$-galactosidase measurement was higher for cells which are pre-exposed to IPTG as compared to the cells which are not exposed (Fig. 1c). These results indicate that the cells with pre-induced lac operon enjoy phenotypic superiority on galactose environment as compared to the ones which are not induced. The biomass was also found to be higher for the pre-induced cells (Fig. 1d).

In order to check whether pre-culture on $1 \mathrm{~g} / \mathrm{L}$ glycerol environment along with $200 \mu \mathrm{M}$ IPTG will provide any benefit for other carbon sources over galactose and lactose. The effect of pre-culture with IPTG on glucose environment was investigated. For this cells were exposed to IPTG on glycerol environment and then grown on glucose environment. Growth kinetics of cells which are pre-induced for lac operon were similar to the ones which are not preinduced (Fig. 2a). The percentage relative decrease in growth rate of the cells which are not exposed to IPTG was very small. It was around $2.5 \%$ (Fig. 2b). The biomass was also almost similar. The growth curve for cells which are pre-induced for lac operon did not show much difference as compared to the cells which are not induced (Fig. 2d). In order to confirm that the phenotypic observation is due to the pre-induced lac operon, the cells were subjected for $\beta$ galactosidase estimation. The cells with pre-induced lac operon showed $\beta$-galactosidase activity while the ones that were not induced showed no activity (Fig. 2c).

The study further investigated the possibility of the counter effect. For this the cells were grown on both glucose
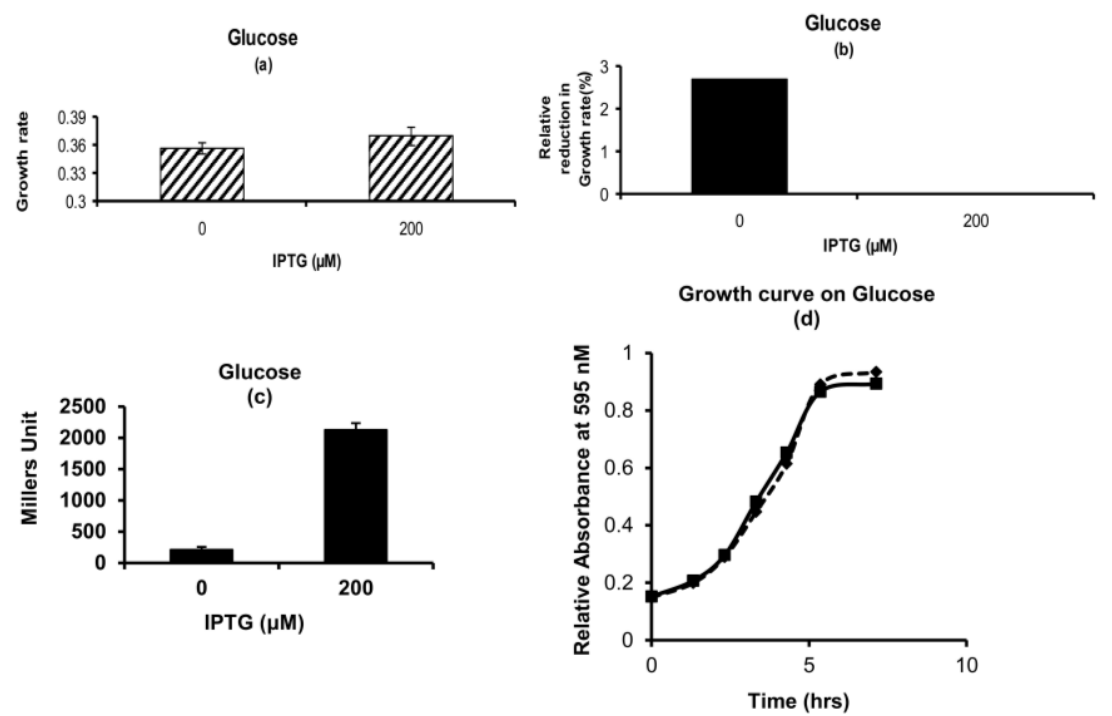

Fig. (2). Phenotypic Growth Properties on Glucose: (a) Growth rate for cells with and without pre-exposure to IPTG. (b) Percentage Relative reduction in growth rate for cells which are not exposed to IPTG in pre-culture as compared to the ones which are exposed to IPTG. (c) $\beta$-galactosidase activity for cells which are exposed to IPTG or not in pre-culture. (d) Growth Curve. Dashed Line with diamond represents the cells which are exposed to $200 \mu \mathrm{M}$ of IPTG in M9 glycerol medium in pre-culture. Solid Line with square represents the cells which are not exposed to IPTG in M9 glycerol medium in pre-culture. Glycerol (1g/L) and Glucose (4g/L) was used in experiment.

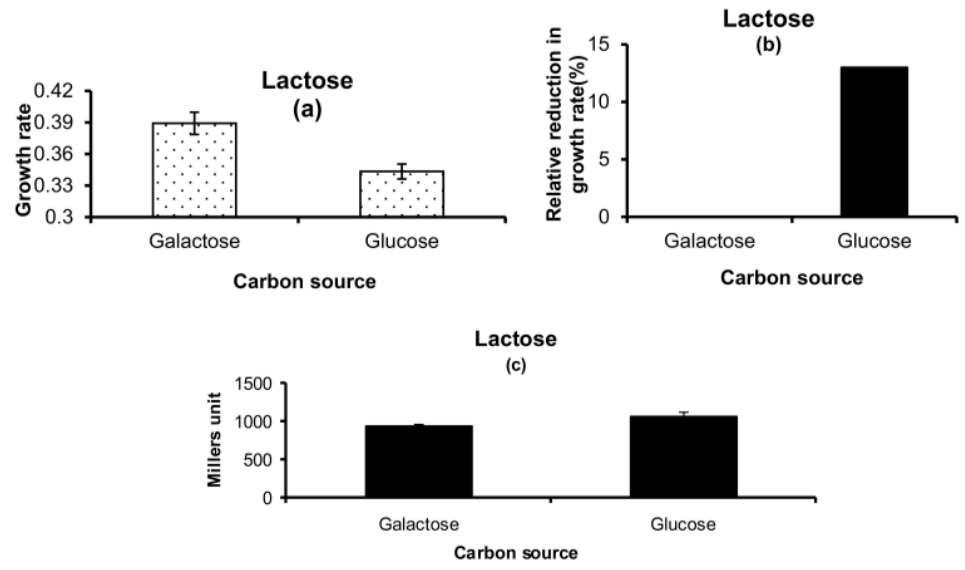

Fig (3). Phenotypic Growth Properties on Lactose: (a) Growth rate on lactose medium for cells exposed to either galactose or glucose as carbon source in pre-culture. (b) Percentage relative reduction in growth rate for cells which are exposed to glucose in pre-culture as compared to the ones which are exposed to galactose in pre-culture on lactcose medium. (c) $\beta$-galactosidase activity for cells. The glucose and galactose written on $\mathrm{X}$-axis represents the carbon source in pre-culture. The concentration of lactose used is $5 \mathrm{mM}$. 
and galactose medium separately in pre-culture and then transferred on lactose medium. The cells which were grown on galactose medium as pre-culture showed higher growth rate on lactose environment as compared to the ones which were grown on glucose as pre-culture (Fig. 3a). The relative reduction in growth rate in percentage was found to be around $14 \%$ for the pre-exposed glucose cells (Fig. 3b). The $\beta$-galactosidase measurement did not differ much for the glucose or galactose pre-grown cells on lactose medium (Fig. 3c).

It is known that pre-induced lac operon provides benefit on lactose environment. It is also known that gene expression is a stochastic process. Therefore, the current study decided to explore the effect of the varied strength of pre-induced lac operon on the growth properties on varying lactose environment. Moreover, it is also known that cost or the effect of unnecessary gene expression is more in the early exponential phase as compared to late exponential phase and is also dependent on substrate concentration [19]. Therefore, the current work also investigated the effect of pre-induced lac operon on lactose environment in EEP and LEP at $1 \mathrm{mM}$ and $5 \mathrm{mM}$ of lactose.

In the EEP the growth rate showed increasing trend with increasing strength of IPTG used in the pre-culture on $1 \mathrm{mM}$ of lactose (Fig. 4a). The growth rate for the cells which are pre-induced with lac operon was more on lactose environment as compared to the ones which are not preinduced. During LEP the growth rate of the cells which are pre-induced with IPTG was more than the cells which are not pre-induced or induced with very low concentration of IPTG (Fig. 4b).

The $\beta$-galactosidase measurement showed increasing trend till $25 \mu \mathrm{M}$ of IPTG used in pre-culture and thereafter it saturates (Fig. 4c). Similar trend was also observed in the late exponential phase however with higher Millers unit (Fig. 4d).

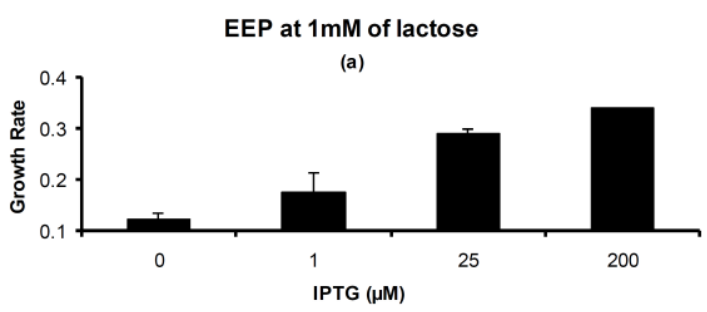

EEP at $1 \mathrm{mM}$ of Lactose

(c)

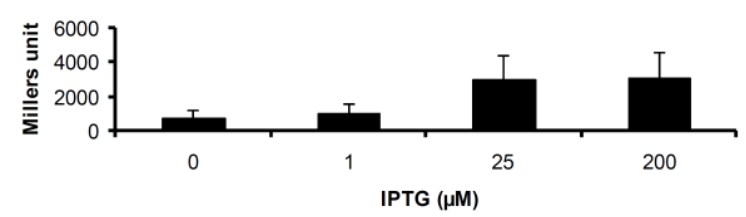

At $5 \mathrm{mM}$ of lactose concentration in the EEP the growth rate followed an increasing trend till $25 \mu \mathrm{M}$ of IPTG concentration and thereafter it saturates (Fig. 5a). In LEP the growth phenotype was not observed as in the EEP. At $5 \mathrm{mM}$ of lactose concentration in LEP the growth rate was slightly higher for 0 and $1 \mu \mathrm{M}$ concentrations of IPTG than 25 and $200 \mu \mathrm{M}$ of IPTG used in the pre-culture (Fig. 5b). In the EEP $\beta$-galactosidase measurement showed increasing trend till $25 \mu \mathrm{M}$ of IPTG concentration after which it saturates (Fig. 5c). In the LEP the $\beta$-galactosidase measurement was almost similar for all cells irrespective of whether they are pre-induced or not (Fig. 5d). These results pointed towards the fact that strength of substrate plays an important role in influencing the effect of pre-induced lac operon on lactose environment. This effect also depends on the growth stage i.e. whether the cells are in EEP or in LEP.

\section{DISCUSSION}

The focus of this study was to characterize the specificity of pre-induced lac operon along with the factors that regulate its activity. The phenotypic superiority of the cells with preinduced lac operon over non induced cells on galactose environment could be explained by the fact that pre-induced lac operon might be helping in galactose metabolism. Preinduced lac operon could help in the galactose uptake which in turn could help in the galactose metabolism. This is the first report of its kind which suggests that pre-induced lac operon is providing benefit on galactose environment. The $\beta$ galactosidase expression for cells which are pre-induced with IPTG is due to the fact that $\beta$-galactosidase is already produced in their pre-culture state and is sustained in galactose environment as it is a very stable protein [21].

This phenotype superiority on galactose environment due to pre-induced lac operon was not extended to glucose environment. This showed that effect of IPTG in pre-culture is restricted to lactose specifically and galactose non-

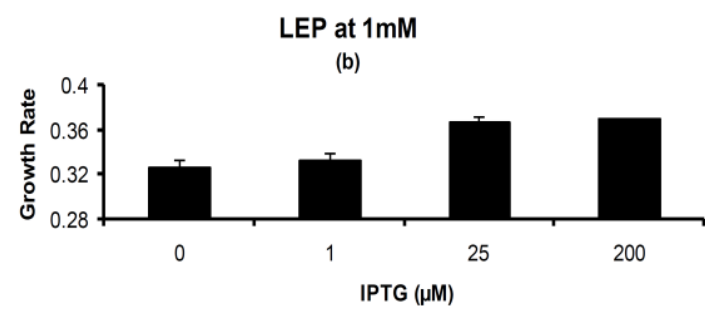

LEP at $1 \mathrm{mM}$ of lactose

(d)

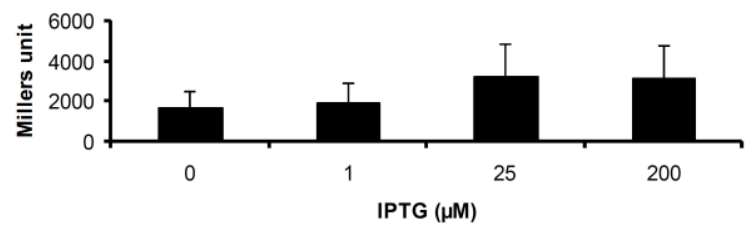

Fig. (4). Phenotypic Properties for Pre-induced Lac Operon on 1 mM of Lactose. (a) \& (b) Growth rate for cells exposed to different concentrations of IPTG in pre-culture on lactose medium in entry into Early exponential Phase and Late Exponential Phase. (c) \& (d) $\beta$ galactosidase activity for cells exposed to different concentrations of IPTG in pre-culture on lactose medium in EEP and LEP. Concentrations of IPTG on X-axis represent the concentration of IPTG to which cells are exposed in pre-culture. Glycerol (1g/L) was used in pre-culture. 

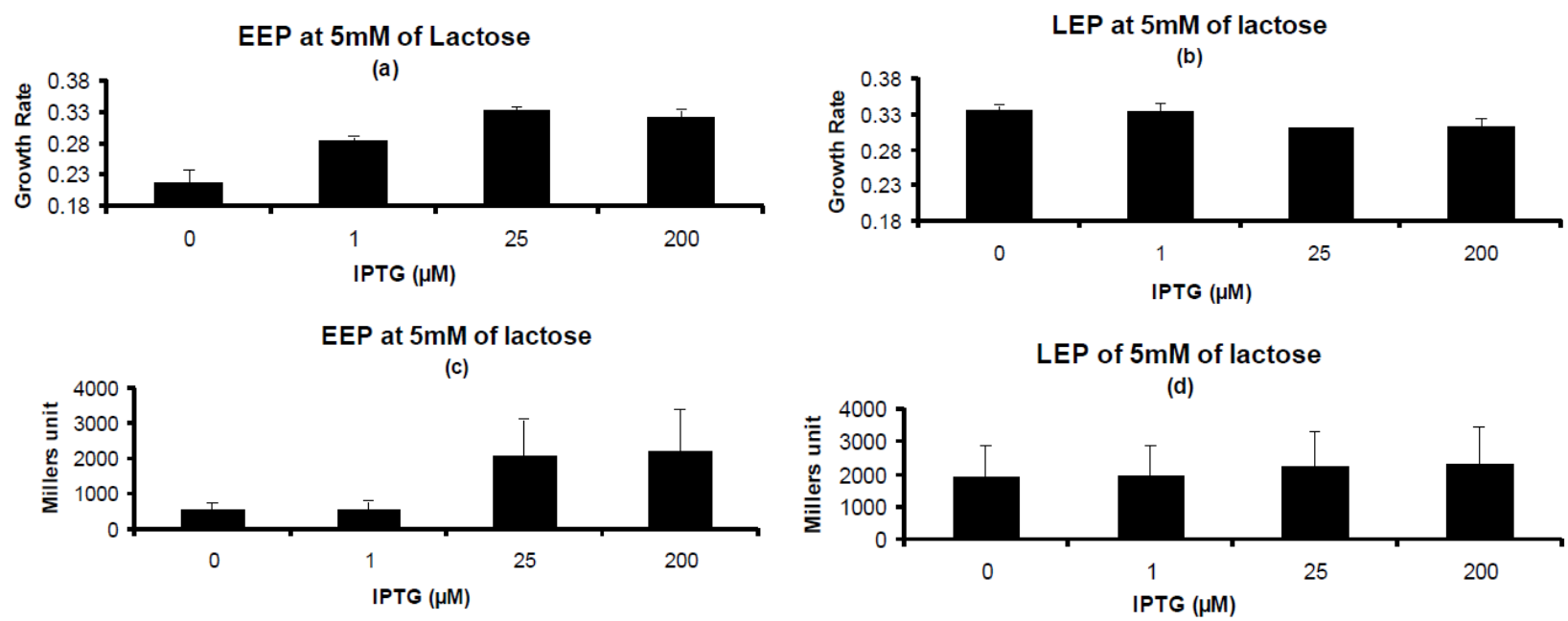

Fig (5). Phenotypic Properties for Pre-induced Lac Operon on 5 mM of Lactose. (a) \& (b) Growth rate for cells exposed to different concentrations of IPTG in pre-culture on lactose medium in entry into Early exponential Phase and Late Exponential Phase. (c) \& (d) $\beta$ galactosidase activity for cells exposed to different concentrations of IPTG in pre-culture on lactose medium in EEP and LEP. Concentrations of IPTG on X-axis represent the concentration of IPTG to which cells are exposed in pre-culture. Glycerol (1g/L) was used in pre-culture.

specifically. The $\beta$-galactosidase measurement observed in glucose environment for cells with pre-induced lac operon is due to the fact that $\beta$-galctosidase produced in the pre-culture state is sustained. Glucose does not activate lac operon [22].

The observation that cells which are pre-cultured on galactose provide phenotypic benefit on lactose environment as compared to the cells which are pre-cultured on glucose (Fig. 3a, 3b), could be due to the fact that exposure to galactose in pre-culture is providing some sort of benefit on lactose environment in terms of lactose utilization. Lactose is cleaved into glucose and galactose. Galactose neither represses lac operon nor induces lac operon [22, 23]. Glucose does not activate lac operon. In case of glucose, it is not the repression but an absence of transcription activation by the CRP-cAMP complex which increases the frequency of RNA-Polymerase binding. The $\beta$-galactosidase activity was almost similar for both types of cells due to the fact that neither galactose nor glucose is the inducer for lac operon and now the cells are exposed to lactose which is the inducer for lac operon.

Varying strength of pre-induced lac operon execute its effect in EEP at $1 \mathrm{mM}$ and $5 \mathrm{mM}$ of lactose. The varying concentration of IPTG used in the pre-culture induces the lac operon to different extent. Higher the $\beta$-galactosidase production, faster will be the utilization of lactose.

In entry into late exponential phase at $1 \mathrm{mM}$ of lactose the phenotypic properties observed was slightly different. In LEP apart from strength of induction of lac operon, lactose might be playing a major role in determining the fate of the cell, which could not be the case in EEP.

Substrate plays an important role in determining the fate of cells with pre-induced lac operon. This is evident from the observation that effect of pre-induced lac operon was not observed in LEP at $5 \mathrm{mM}$ of lactose. This is further supported by the fact that $\beta$-galactosidase production was almost similar for the cells in LEP at $5 \mathrm{mM}$ of lactose, exposed to different concentrations of IPTG in pre-culture. In LEP high concentration of lactose nullifies the effect of pre-induced lac operon.

In summary the result shows the phenotypic advantage of cells exposed to IPTG in pre-culture or pre-induced lac operon on galactose environment. This is the first report providing evidence for induced lac operon providing benefit on galactose environment other than lactose. This benefit is restricted only to lactose and galactose. Moreover, the cells exposed to galactose in pre-culture also enjoy higher growth rate on lactose milieu as compared to the ones which are exposed to glucose. Strength of pre-induced lac operon plays an important role in determining phenotypic response on entering into Early Exponential Phase and Late Exponential Phase on lactose environment. Lactose concentration also plays an important role in determining the effect of genetic state of the cells, due to pre-induced lac operon on growth physiology on lactose environment. The present studies will help in the better understanding of the relation of gene expression and growth physiology.

\section{CONFLICT OF INTEREST}

The author declares that there exists no conflict of interest.

\section{ACKNOWLEDGMENTS}

Pushkar Malakar is very thankful to Council of Scientific and Industrial Research (CSIR) and IIT Bombay for all financial support. He is the recipient of Senior Research Fellowship from CSIR. He is indebted to Manjula Reddy of CCMB, Hyderabad, India for giving MG1655 Strain as generous gift. Pushkar Malakar is grateful to Professor K.V.Venkatesh of IITB for providing the resources and space for experiments. The author thanks Anbumathi for giving valuable comments on the paper. 


\section{REFERENCES}

[1] Kandhai MC, Reij MW, Grognou C et al. Effects of preculturing conditions on lag time and specific growth rate of enterobacter sakazakii in reconstituted powdered infant formula. Appl Environ Microbiol 2006;72:2721-9.

[2] Acar M, Becskei A, Van Oudenaarden A. Enhancement of cellular memory by reducing stochastic transitions. Nature 2005;435:22832 .

[3] Malakar P,Venkatesh KV.Characterization of burden on growth due to the nutritional state of media and pre-induced gene expression. Arch Microbiol. 2005; 195: 291-5.

[4] Hansen LH, Knudsen S, Sørensen SJ. The effect of the lacY gene on the Induction of IPTG inducible promoters, Studied in Escherichia coli and Pseudomonas fluorescens. Curr Microbiol 1998; 36: 341-7.

[5] Jacob F, Monad J. Genetic regulatory mechanisms in the synthesis of proteins. J Mol Biol 1961; 3: 318-56.

[6] Scott M, Gunderson CW, Mateescu EM et al. Interdependence of cell growth and gene expression. Original Conseq Sci 2010; 330: 1099-102.

[7] Klumpp S, Zhang Z, Hwa T. Growth rate-dependent global effects on gene expression in bacteria. Cell. 2009; 139: 1366-75.

[8] Kuo J-T, Chang Y-J, Tseng C-P. Growth rate regulation of lac operon expression in Escherichia coli is cyclic AMP dependent. FEBS Lett 2003; 553: 397-402.

[9] Pedersen S, Bloch PL, Reeh S, Neidhardt FC. Patterns of protein synthesis in E. coli: a catalog of the amount of 140 individual proteins at different growth rates. Cell 1978;14:179-90.

[10] Gonzalez R, Tao H, Shanmugam KT, et al. Global gene expression differences associated with changes in glycolytic flux and growth rate in Escherichia coli during the fermentation of glucose and xylose. Biotechnol Prog 2002; 18: 6-20.

[11] Gourse RL, de Boer HA, Nomura M. DNA determinants of rRNA synthesis in E. coli: growth rate dependent regulation, feedback inhibition, upstream activation, antitermination. Cell 1986; 44: 197205.
[12] Emilsson V, Kurland CG. Growth rate dependence of transfer RNA abundance in Escherichia coli. EMBO J 1990; 9: 4359-66.

[13] Gourse RL, Gaal T, Bartlett MS, et al.Rrna transcription and growth rate-dependent regulation of ribosome synthesis in Escherichia coli. Annu Rev Microbiol 1996; 50: 645-77.

[14] Raj A, van Oudenaarden A.Nature, Nurture, or chance: stochastic gene expression and its consequences. Cell 2008; 135: 216-26.

[15] Malakar P, Singh V, Karmakar R et al.Effect on beta-galactosidase synthesis and burden on growth of osmotic stress in Escherichia coli. Springer Plus 2014; 3: 748 .

[16] Malakar P, Venkatesh K. Effect of substrate and IPTG concentrations on the burden to growth of Escherichia coli on glycerol due to the expression of Lac proteins. Appl Microbiol Biotechnol 2007; $1-7$.

[17] Malakar P. Characterization of cost with respect to nutritional upshift in the media composition along with sublethal doses of transcriptional and translational inhibitor. Arch Microbiol 2014;16.

[18] Malakar P,Venkatesh KV. GAL regulon of Saccharomyces cerevisiae performs optimally to maximize growth on galactose. FEMS Yeast Res 2013; 14(2): 346-56.

[19] Shachrai I, Zaslaver A, Alon U, et al. Cost of Unneeded Proteins in E. coli Is Reduced after Several Generations in Exponential Growth. Mol Cell 2010; 38: 758-67.

[20] Samaluru H, SaiSree L. Reddy M. Role of SufI (FtsP) in cell division of Escherichia coli: evidence for its involvement in stabilizing the assembly of the divisome. J Bacteriol 2010; 189: 8044-52.

[21] Yoshioka S, Aso Y, Izutsu K-i,Terao T. Stability of $\beta$ galactosidase, a model protein drug, is related to water mobility as measured by $<$ sup $>17</$ sup $>$ O nuclear magnetic resonance (NMR). Pharm Res 1993; 10: 103-8.

[22] Griffiths AJ, Miller JH, Suzuki DT et al. WM Catabolite repression of the lac operon: positive control. $7^{\text {th }}$ ed. New York; W.H. Freeman 2010.

[23] Leder IG Perry JW. Galactose stimulation of $\beta$-galactosidase induction in galactokinaseless mutants of Escherichia coli. J Biol Chem 1967; 242: 457-62. 